\title{
Nanopencil as a wear-tolerant probe for ultrahigh density data storage
}

\author{
Noureddine Tayebi, ${ }^{1}$ Yoshie Narui, ${ }^{2}$ Robert J. Chen, ${ }^{1}$ C. Patrick Collier, ${ }^{2, a)}$ \\ Konstantinos P. Giapis, ${ }^{2}$ and Yuegang Zhang ${ }^{1, b)}$ \\ ${ }^{1}$ Intel Corporation, 2200 Mission College Boulevard, Santa Clara, California 95054, USA \\ ${ }^{2}$ Division of Chemistry and Chemical Engineering, California Institute of Technology, Pasadena, \\ California 91125, USA
}

(Received 17 July 2008; accepted 19 August 2008; published online 11 September 2008)

\begin{abstract}
A dielectric-sheathed carbon nanotube probe, resembling a "nanopencil," has been fabricated by conformal deposition of silicon-oxide on a carbon nanotube and subsequent "sharpening" to expose its tip. The high aspect-ratio nanopencil probe takes advantage of the small nanotube electrode size, while avoiding bending and buckling issues encountered with naked or polymer-coated carbon nanotube probes. Since the effective electrode diameter of the probe would not change even after significant wear, it is capable of long-lasting read/write operations in contact mode with a bit size of several nanometers. (C) 2008 American Institute of Physics. [DOI: 10.1063/1.2981641]
\end{abstract}

Small bit size and fast data rate have made probe-based seek-and-scan data storage systems the ideal candidate for future ultrahigh density nonvolatile memories. ${ }^{1-4}$ In such systems, a scanning probe (or an array of probes) is used to write and read data on nonvolatile media; the bit size depends mainly on the radius of the probe tip. Recent studies have shown that the field confinement of a nanometer-scale tip radius could locally reverse the polarization of a ferroelectric thin film and create domain size as small as a few nanometers in diameter. ${ }^{1,2,5}$ Such technology could realize storage density of $10 \mathrm{Tbits} /$ in. $^{2}{ }^{2}$ The high-speed contact mode operation in practical devices, however, can cause rapid mechanical wear and dulling of conventional scanning probe tips, thus decreasing resolution. Due to their strong mechanical and wear-resistant properties, carbon nanotubes have attracted interest as probe tips in scanning probe lithography and data storage. ${ }^{7-9}$ The combination of their small diameter and high aspect ratio enable carbon nanotubes to retain their read/write resolution even after significant wear. However, carbon nanotubes are prone to bending and buckling, which can be mitigated partially only with short nanotubes $\left(\sim 100 \mathrm{~nm}\right.$ in length) ${ }^{7-9}$ Such constraints hinder the use of carbon nanotube probes in practical data storage devices where contact mode is required for high data rate, and longer carbon nanotubes are needed to outlast the storage device lifetime. Here we demonstrate dielectric-coated carbon nanotube probes of high aspect ratio with wear resistance and stiffness suitable for long-lasting read/write operations in contact mode. Moreover, such probes are capable of writing bit sizes with radii as small as $6.8 \mathrm{~nm}$ on ferroelectric films, and thus allowing for a storage density of $1 \mathrm{Tbit} / \mathrm{in}^{2}$

Long $(1-2 \mu \mathrm{m})$ single-walled carbon nanotubes (SWNTs) (or their bundles of 5-10 $\mathrm{nm}$ in diameter) are first attached to metal-coated scanning probe tips. ${ }^{10,11}$ This is achieved by imaging isolated and vertically aligned SWNTs grown on a $\mathrm{SiO}_{2}$ film using the metal-coated tips, during which the isolated SWNTs attach to the tips. Note that the

\footnotetext{
${ }^{\text {a) }}$ Present address: Oak Ridge National Laboratory, Center for Nanophase Materials Sciences, Oak Ridge, TN 37831.

${ }^{b)}$ Author to whom correspondence should be addressed. Present address: The Molecular Foundry, Lawrence Berkeley National Laboratory, Berkeley, California 94720. Electronic mail: yzhang5@1bl.gov.
}

attached SWNTs can either be metallic or semiconducting in nature because of the lack of a reliable method for nanotube type selection. However, this is not a problem for the current study because all chemical vapor deposited semiconducting SWNTs are $p$-doped when exposed to air. In the bundle cases, the metallic SWNTs in the bundle normally dominate electric conductance. The probe system is then coated with a conformal 65-nm-thick $\mathrm{SiO}_{x}$ layer using a plasma-assisted chemical vapor deposition at room temperature. ${ }^{11,12}$ The resulting $\mathrm{SWNT} / \mathrm{SiO}_{x}$ composite structure resembles a nanopencil, as shown in Fig. 1(a). When exposed, the SWNT core serves as a thin electrode, while the thick $\mathrm{SiO}_{x}$ sheath improves its mechanical strength during read/write operations [Fig. 1(b)]. Additionally, the coating reinforces the electrical and mechanical contact between the base probe and SWNT, and thus prevents the tube detachment under contact mode scanning.

To expose the insulated SWNT electrode, the nanopencil is "sharpened" by repetitively scanning a $20 \times 20 \mu \mathrm{m}^{2}$ area of a diamond surface in contact mode at a speed of $100 \mu \mathrm{m} / \mathrm{s} .^{11}$ This is performed using the conductive AFM module of an Asylum Research MFP-3D scanning probe microscope operating under contact mode with a $5 \mathrm{nN}$ applied force. By simultaneously recording the height and current signals, the sharpening process is monitored in real time by applying a $0.5 \mathrm{~V}$ bias between the probe and the conductive diamond sample until a significant current is detected, as shown in Fig. 2. Figure 3 shows a $1 \times 1 \mu \mathrm{m}^{2}$ height and current distribution images, and $I-V$ curve taken after electrode exposure. The resistance $R=3.6 \mathrm{M} \Omega$ corresponds to

(a)
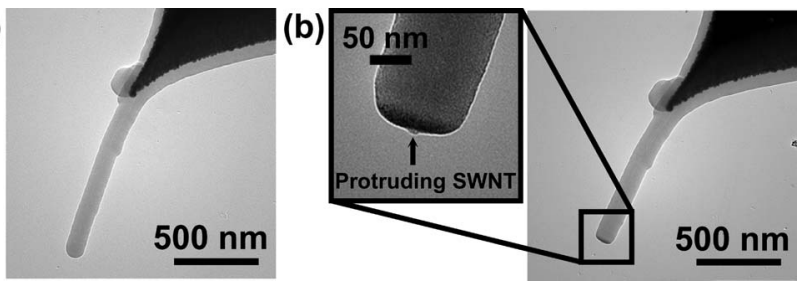

FIG. 1. TEM images of the nanopencil. (a) Nanopencil before SWNT electrode exposure (initial length: $980 \mathrm{~nm}$ ). (b) Nanopencil after electrode exposure (length: $870 \mathrm{~nm}$ ). The inset shows the clean SWNT electrode protruding from the $\mathrm{SiO}_{x}$ coating. 

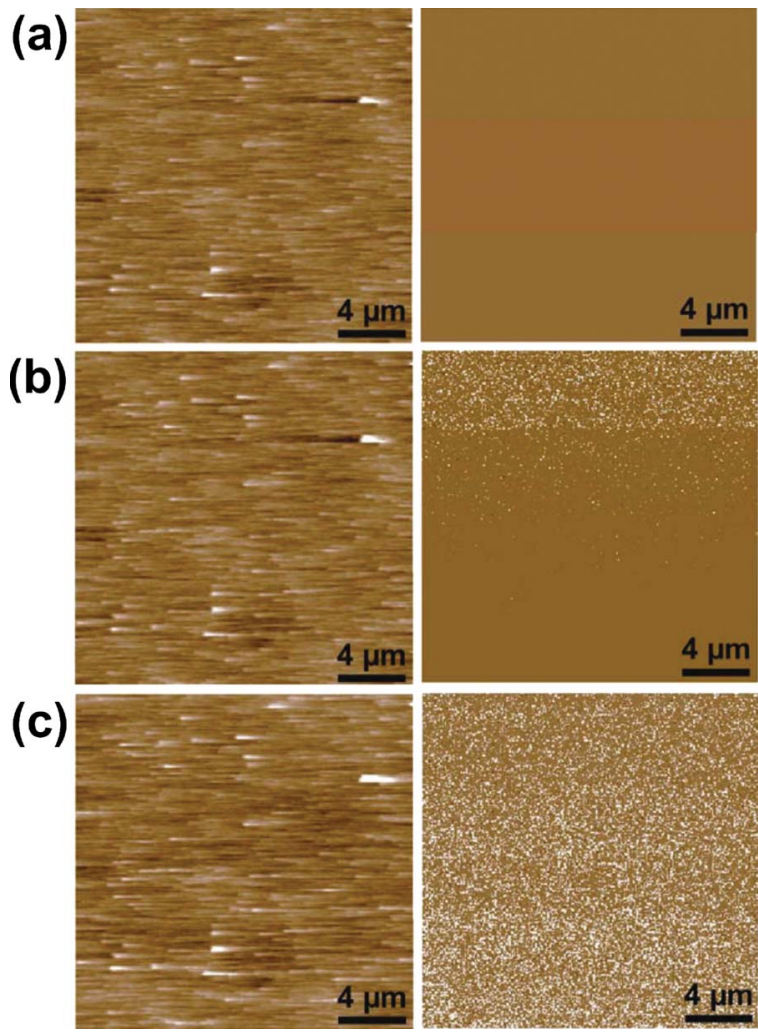

FIG. 2. (Color online) Real time monitoring of the nanopencil sharpening process by conductive AFM. [(a)-(c)] $20 \times 20 \mu \mathrm{m}$ height and current distribution images (left to right) of the conductive diamond film recorded during the experiment to expose the SWNT electrode. (a) Frame 1 (nanopencil scanning down). No current flow between pencil and diamond film. (b) Frame 11 (nanopencil scanning up). Current starts to flow indicating that the SWNT electrode started to be exposed. (c) Frame 17 (nanopencil scanning up). Current is fully flowing indicating that the SWNT electrode is fully exposed.

mainly contact resistance between the nanopencil and the diamond film. The SWNT exposure is also confirmed using a Phillips EM420 transmission electron microscope (TEM) operating at $120 \mathrm{kV}$, as shown in Fig. 1(b). Note the cleanliness and protrusion of the SWNT electrode during the sharpening process.

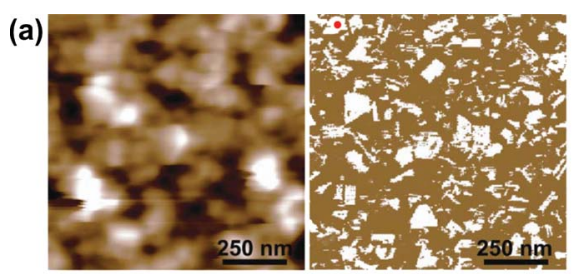

(b)

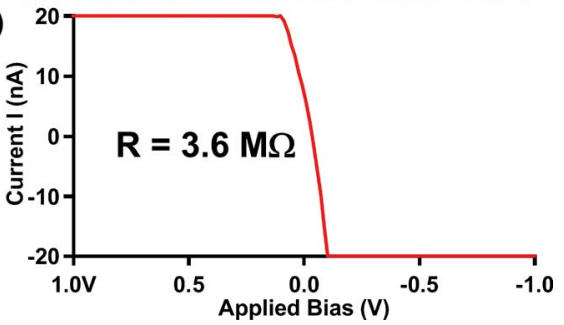

FIG. 3. (Color online) (a) $1 \times 1 \mu \mathrm{m}$ height (left) and current (right) distribution images on the conductive diamond film taken after electrode exposure (nanopencil scanning up). (b) $I-V$ curve taken at the location indicated by the red dot on the current distribution image. The resistance $R$ $=3.6 \mathrm{M} \Omega$ corresponds to mainly contact resistance between the nanopencil and the diamond film. (a)
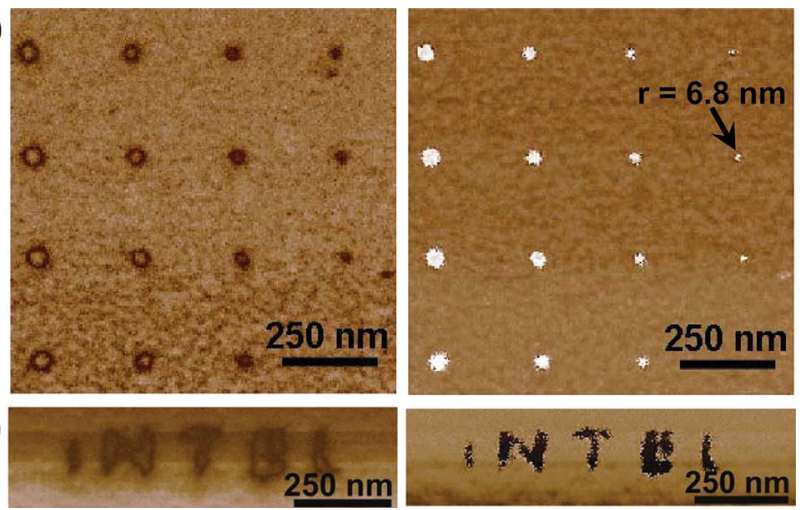

(b)

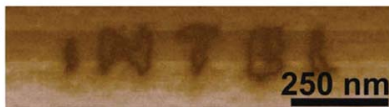

FIG. 4. (Color online) PFM amplitude (right) and phase (left) images of PZT surfaces with ferroelectric domains formed by applying electrical pulses for $100 \mu$ s to the PZT surfaces through the conductive nanopencils. (a) Small ferroelectric domains formed by applying 7-10 V pulses. The pulse potential decreases by a $1 \mathrm{~V}$ increment (left to right). Measurements from the second top row of the dots in the phase image give dot radii of 21.5, 17.5, 15.5, and $6.8 \mathrm{~nm}$. (b) Ferroelectric domain patterns written by another nanopencil probe with a series of $6 \mathrm{~V}$ pulses.

The nanopencil is then used to write inverted domain dots on a 50-nm-thick ferroelectric film of lead zirconate titanate (PZT) epitaxially grown on (001) $\mathrm{SrTiO}_{3}$ film, ${ }^{13}$ which is initially polarized in an upward direction and placed on a grounded electrode. ${ }^{1-3,11}$ The ferroelectric domains are imaged using the same nanopencil under piezoresponseforce microscopy (PFM) mode of the Asylum Research AFM system. The writing of a $4 \times 4$ dot matrix is accomplished by applying short electrical pulses $(7-10 \mathrm{~V})$ with a pulse width of $100 \mu$ s to the PZT surface through the conductive nanopencil core. Such pulses create highly concentrated downward electric fields underneath the nanopencil that, in turn, flip the polarization of local PZT film volumes and form downward polarization domains that can be used as bits for data storage. ${ }^{1,5,11}$ Domain dots with radii as small as $6.8 \mathrm{~nm}$ could be reliably written, as shown in Fig. 4(a). Such domain sizes can easily allow for a storage density of $1 \mathrm{Tbit} / \mathrm{in} .{ }^{2}$. With precise control of the nanopencil position on the PZT surface, more complex patterns can also be written, as shown in Fig. 4(b).

The propensity for the nanopencil probes to buckle or bend during scanning can be characterized by the Euler buckling force and the lateral spring constant, estimated to be $14.5 \mu \mathrm{N}$ and $2.94 \mathrm{~N} / \mathrm{m}$, respectively, for a $1 \mu \mathrm{m}$ long nanopencil. ${ }^{11}$ The estimated buckling force is three orders of magnitude larger than the $5 \mathrm{nN}$ applied normal force during typical read and write operations, and about 14 times larger than that previously reported for $1-3 \mu \mathrm{m}$ multiwalled carbon nanotube (MWNT) probes coated with parylene. ${ }^{14}$ We have also calculated the lateral spring constant ${ }^{11,15}$ of the MWNT/polymer probe in Ref. 14. The result is 150 times smaller than our SWNT/SiO ${ }_{x}$ nanopencil. ${ }^{11}$ Thus our nanopencil probes can achieve the demanded mechanical stability for precise read and write operations that rely on the vertical piezoresponse of the system and can be greatly affected by the bending and buckling of the probe. Moreover, by modeling the nanopencil-PZT film contact as a contact between two rough surfaces, ${ }^{11,16,17}$ we estimate the wear rate of the nanopencil on the PZT film to be $4.36 \times 10^{-3} \mathrm{~nm} / \mathrm{s}$ at a scanning speed of $50 \mathrm{~mm} / \mathrm{s}$. At this wear rate, a $1 \mu \mathrm{m}$ long nanopencil could survive $11.5 \mathrm{~km}$ scanning distance without 
losing read/write resolution, which exceeds the requirement of most common data storage applications.

In summary, high aspect-ratio SWNT-based nanopencil probes for ultrahigh density nonvolatile memories have been fabricated by conformal deposition of silicon-oxide on a carbon nanotube and subsequent sharpening to expose its tip. Such dielectric-reinforced probes take advantage of the small SWNT electrode size, while avoiding bending and buckling issues encountered with naked or polymer-coated SWNT probes. Even under high wear rates, such probes are capable of long-lasting high resolution read/write operations in contact mode with a bit size of several nanometers.

${ }^{1}$ Y. Cho, S. Hashimoto, N. Odagawa, K. Tanaka, and Y. Hiranaga, Nanotechnology 17, S137 (2006).

${ }^{2}$ Y. Cho, S. Hashimoto, N. Odagawa, K. Tanaka, and Y. Hiranaga, Appl. Phys. Lett. 87, 232907 (2005).

${ }^{3}$ C. H. Ahn, J.-M. Triscone, N. Archibald, M. Decroux, R. H. Hammond, T. H. Geballe, O. Fischer, and M. R. Beasley, Science 269, 373 (1995).

${ }^{4}$ P. Vettiger, M. Despont, U. Drechsler, U. Durig, W. Haberle, M. I. Lutwyche, H. E. Rothuizen, R. Stutz, R. Widmer, and G. K. Binnig, IEEE
Trans. Nanotechnol. 1, 39 (2002).

${ }^{5}$ A. Gruverman and S. V. Kalinin, J. Mater. Sci. 41, 107 (2006).

${ }^{6}$ B. J. Rodriguez, R. J. Nemanich, A. Kingon, A. Gruverman, S. V. Kalinin, K. Terabe, X. Y. Liu, and K. Kitamura, Appl. Phys. Lett. 86, 012906 (2005).

${ }^{7}$ K. Ishikawa, K. Honda, and Y. Cho, Nanotechnology 18, 084015 (2007).

${ }^{8}$ E. Cooper, S. R. Manalis, H. Fang, H. Dai, K. Matsumoto, S. C. Minne, T. Hunt, and C. F. Quate, Appl. Phys. Lett. 75, 3566 (1999).

${ }^{9}$ H. Dai, N. Franklin, and J. Han, Appl. Phys. Lett. 73, 1508 (1998).

${ }^{10}$ J. H. Hafner, C. L. Cheung, T. H. Oosterkamp, and C. M. Lieber, J. Phys. Chem. B 105, 743 (2001).

${ }^{11}$ See EPAPS Document No. E-APPLAB-93-033837 for the detailed experiment and calculation methods. For more information on EPAPS, see http:// www.aip.org/pubservs/epaps.html.

${ }^{12}$ M. J. Esplandiu, V. G. Bittner, K. P. Giapis, and C. P. Collier, Nano Lett. 4, 1873 (2004).

${ }^{13}$ D. M. Kim, C. B. Eom, V. Nagarajan, J. Ouyang, R. Ramesh, V. Vaithyanathan, and D. G. Schlom, Appl. Phys. Lett. 88, 142904 (2006).

${ }^{14}$ A. Patil, J. Sippel, G. W. Martin, and A. G. Rinzler, Nano Lett. 4, 303 (2004).

${ }^{15}$ N. R. Wilson and J. V. Macpherson, Nano Lett. 3, 1365 (2003).

${ }^{16}$ N. Tayebi and A. A. Polycarpou, J. Appl. Phys. 98, 073528 (2005).

${ }^{17}$ B. Bhushan, Introduction to Tribology (Wiley, New York, 2002). 